\title{
Significance of NF-kappaB signaling and PARP1 activity in the TNF-induced inhibition of PHEX gene expression in human osteoblasts*
}

\author{
Urszula Kedzierska1, Lukasz Banasiak², Pawel R. Kiela³ and Pawel M. Majewski ${ }^{1 凶}$ \\ 1Department of Animal Physiology, Faculty of Biology, University of Warsaw, Warsaw, Poland; 2Department of Molecular Phylogenetics and Evo- \\ lution, Institute of Botany, Faculty of Biology and Biological and Chemical Research Centre, University of Warsaw, Warsaw, Poland; ${ }^{3}$ Departments \\ of Pediatrics and Immunobiology, Steele Children's Research Center, University of Arizona, Tucson AZ 85724, USA
}

\begin{abstract}
Although loss of bone mineral density is a common symptom of chronic inflammatory diseases, its mechanisms are still poorly understood. The PHEX gene encodes a Zn-endopeptidase expressed in osteoblasts and contributes to bone mineralization. Data derived from rodents has indicated co-repression of the PHEX gene by the NF-KB pathway and poly(ADP-ribose) polymerase 1 (PARP1). The aim of this study was to determine the molecular mechanism involved in TNF-mediated downregulation of PHEX expression in human osteoblasts and human osteosarcoma cell line. We observed that activation of the NF-KB pathway by TNF was manifested as a nuclear increase in RELA and NFKB1 heterodimer. We found that TNF reduced PHEX expression and the proteasome inhibitor reversed this effect in osteosarcoma cell line. Contrary to the effects seen in rodents, inhibition of PARP1 enzymatic activity did not significantly reverse the effect of TNF on the human PHEX gene expression. EMSA studies showed that the number of adenines in the PHEX proximal promoter is crucial for the transcription factors' interactions within that region. The obtained results support the hypothesis indicating the existence of a molecular mechanism of gene repression that involves a poly adenine-rich region of the proximal gene promoters and PARP1 transcriptional activity.
\end{abstract}

Key words: osteoblast, tumor necrosis factor, NF-KB, PARP1

Received: 18 June, 2018; revised: 23 September, 2018; accepted: 15 October, 2018; available on-line: 16 November, 2018

Qe-mail: pmajew@biol.uw.edu.pl

*Preliminary report: These results were presented at the XXI International symposium "Molecular and physiological aspects of regulatory processes of the organism", Krakow, Poland (14-15 Jun 2012) and at 2nd Joint Meeting of the International Bone and Mineral Society and the Japanese Society for Bone and Mineral Research, Kobe, Japan (27 May-1 Jun 2013).

Abbreviations: BMD, bone mineral density; FGF-23, fibroblast growth factor 23; Fra1, fos-related antigen $1 ; \mathrm{HOb}$, human bone osteoblasts; IBD, inflammatory bowel diseases; MG-63, human osteosarcoma cells; NF-KB, nuclear factor-kappa B pathway; NFKB1, nuclear factor kappa B subunit 1; NFKB2, nuclear factor kappa B subunit 2; OBs, osteoblasts; OCs, osteoclasts; PARP1, poly(ADP-ribose) polymerase 1: PARG, poly(ADP-ribose) glycohydrolase ; PARyl, poly(ADP)-ribosylation; PHEX, phosphate regulating endopeptidase homolog X-linked; $\mathrm{Pi}$, inorganic phosphate; PolyA, adenine-reach region of the promoter; REL, REL proto-oncogene, NF-KB subunit; RELA, RELA proto-oncogene, NF-KB subunit; TNF, tumor necrosis factor; UMR-106, rat osteosarcoma cell line; XLH, X-linked hypophosphatemic rickets

\section{INTRODUCTION}

Decreased bone mineral density (BMD) is common in patients with chronic inflammatory conditions associated with increased levels of inflammatory mediators (Viswanathan \& Sylvester, 2008). Osteoporosis, characterized by progressive loss of $\mathrm{BMD}$, leads to an increased risk of fracture and is one of the most common bone diseases (Sponholtz et al., 2014). The lifetime risk of an osteoporotic fracture in the United States is estimated at almost $40 \%$ for women and approximately $13 \%$ for men (Johnell \& Kanis, 2005). Moreover, osteoporosis-related fractures are associated with considerable morbidity and mortality in older people (Johnell \& Kanis, 2004; Johnell et al., 2004). Both in vivo and in vitro studies have demonstrated that pro-inflammatory cytokines, such as interleukin-6 (IL-6), interleukin-1 beta (IL-1 $\beta$ ), and tumor necrosis factor (TNF), influence the age- and estrogen-related decrease in BMD (Sponholtz et al., 2014). These immune factors have been shown to promote the proliferation, activity, and survival of osteoclasts while inhibiting the survival of osteoblasts (Clowes, Riggs, \& Khosla, 2005).

The changes in the bone matrix are associated with the activity of three major cell types: bone forming osteoblasts (OBs) and osteocytes, and bone resorbing osteoclasts (OCs) (Teti \& Zallone, 2009). The balance between the two opposite processes is the basis for maintaining bone homeostasis and may be easily upset, leading to a decrease in BMD. This also occurs during chronic autoinflammatory conditions, such as inflammatory bowel diseases (IBD). IBD patients have increased levels of proinflammatory cytokines, including TNF, and altered bone homeostasis (Viswanathan \& Sylvester, 2008). The pathogenesis of osteopenia and osteoporosis in IBD has been suggested to result from altered rates of bone formation and resorption secondary to a multifactorial mechanism (Ghishan \& Kiela, 2011), which includes two major categories: (1) poor nutritional status and malabsorption and (2) inflammation with IBD-associated inflammatory cytokines affecting the bone more directly. Studies conducted on IBD animal models indicated that malabsorption and inflammation are not two independent contributors to IBD-associated BMD loss, and that both inflammation and inflammatory cytokines actively contribute to the abnormalities in intestinal and renal mineral and vitamin absorption (Ghishan \& Kiela, 2011).

The renal-skeletal-gut axis (Kiela \& Ghishan, 2009) is one of the main bone homeostatic mechanisms. This axis regulates systemic levels of calcium $\left(\mathrm{Ca}^{2+}\right)$ and inorganic phosphate $(\mathrm{Pi})$, which are important for systemic homeostasis, including bone formation. PHEX (phosphate-regulating gene with homologies to endopeptidases on the X chromosome), which encodes a $\mathrm{Zn}$-metalloen- 
dopeptidase that is expressed primarily in OBs, osteocytes, and odontoblasts, plays a key role in this process (Kiela \& Ghishan, 2009). A pivotal role of this gene in Pi homeostasis was established in vitamin D-resistant, X-linked hypophosphatemic rickets (XLH) (Du et al., 1996; F. Francis, 1995). Inactivating mutations in the PHEX gene in this familial disorder results in hypophosphatemia, aberrant vitamin D levels, high level of serum alkaline phosphatase and osteomalacia. PHEX gene mutation is associated with decreased expression and activity of the $\mathrm{Na}^{+} / \mathrm{P}_{\mathrm{i}}$ cotransporter NaPi-IIa (NPT2; SLC34A1) responsible for phosphate re-absorption in the renal proximal tubules (Collins \& Ghishan, 1994; Dixon et al., 1998; Drezner, 2000; Hernando, Biber, Forster, \& Murer, 2005; Murer, Hernando, Forster, \& Biber, 2003), which are also pivotal elements of the renal-skeletal-gut axis. PHEX affects renal Pi re-absorption via an indirect action of phosphaturic factors termed phosphatonins. The fibroblast growth factor 23 (FGF-23) is one of the most extensively researched phosphatonin known to inhibit renal $\mathrm{Pi}$ handling and bone mineralization (Kiela \& Ghishan, 2009), and it has been hypothesized that PHEX inactivates FGF-23 via proteolytic degradation (Bowe et al., 2001).

The nuclear factor kappa-light-chain-enhancer of activated $\mathrm{B}$ cells $(\mathrm{NF}-\varkappa \mathrm{B})$ signaling pathway plays a key role in inflammation and immune responses. In OCs NF- $x \mathrm{~B}$ is activated during inflammation leading to increased bone resorption. While the actions of the $\mathrm{NF}-x \mathrm{~B}$ signaling pathway in osteoclasts are well understood (Novack, 2011), less is known about this pathway in OBs, and about its contribution to the BMD regulation. To examine the effects of $\mathrm{NF}-x \mathrm{~B}$ on $\mathrm{OBs}$, transgenic mice expressing a dominant negative form of the inhibitor of nuclear factor kappa B kinase subunit gamma (IKK-DN) were generated (Chang et al., 2009). Young transgenic mice expressing an IKK-DN in OBs had normal numbers of OBs, but increased trabecular bone mass, bone formation rates, and expression of $\mathrm{OB}$ marker genes, although this effect did not persist into adulthood (Chang et al., 2009). Young IKK-DN transgenic mice showed enhanced c-Jun $\mathrm{N}$-terminal kinases (JNK) activation and increased expression of fos-related antigen 1 (Fra1), a protein that was required for the stimulatory effects of $\mathrm{NF}-x \mathrm{~B}$ blockade in OBs.

During chronic inflammation such as IBD, circulating and/or infiltrating lymphocytes and other mononuclear cells produce cytokines that may influence bone metabolism by altering the balance of bone mineral deposition and resorption. Decreased BMD is a common outcome of IBD: 31 to $59 \%$ of adult IBD patients are classified as osteopenic, whereas 5 to $41 \%$ are actually diagnosed with osteoporosis, although the rates of up to $70 \%$ of adult and pediatric IBD patients with low BMD have been reported (Rodriguez-Bores, Barahona-Garrido, \& Yamamoto-Furusho, 2007). In murine experimental colitis, bone PHEX expression was inhibited by TNF and this effect correlated with reduced OBs mineralization in vitro (Uno et al., 2006). TNF treatment and chemically induced colitis decreased murine Phex mRNA expression via a transcriptional mechanism, and the adenine-reach region (PolyA) of the promoter located -116 to -110 nt upstream from the transcriptional start site (TSS) was necessary for the TNF-mediated inhibition (Uno et al., 2006). Further work revealed that murine Phex promoter constitutively binds poly(ADP-Ribose) polymerase 1 (PARP1) in the PolyA region and after TNF treatment RELA proto-oncogene NF- $x$ B subunit (RELA, synonym: p65), a component of the NF- $x \mathrm{~B}$ pathway, is poly(ADP)-ribosylated (PARylated) by PARP1 to increase its activity as a trans-repressor (Majewski, Thurston, Ramalingam, Kiela, \& Ghishan, 2010). Moreover, PARP1 activity was indispensable for the effects of TNF, as demonstrated by a blunted response to the cytokine in the presence of a PARP1 inhibitor or overexpressed poly(ADP-ribose) glycohydrolase (PARG), an enzyme responsible for de-PARylation, and by a complete abrogation of the response to TNF in PARP1 knockout mice (Majewski et al., 2010). These discoveries indicated a novel cooperative mechanism of TNF mediated gene regulation in OBs involving $\mathrm{NF}-x \mathrm{~B}$ and PARP1.

PARP1 protein is involved in numerous important processes at the cellular level, e.g. continuous monitoring of DNA integrity to initiate its repair. Therefore, PARP1 frequently interacts with DNA, and an increase in its positioning close to transcription start sites has also been documented (Krishnakumar et al., 2008). Indeed, recent microarray analysis of the colonic tissue showed extensive transcriptional reprogramming (Larmonier et al., 2016), thus confirming important role of PARP1 as a transcriptional modulator. Both, PARP1 and $\mathrm{NF}-x \mathrm{~B}$ pathway have been suggested to play a crucial role in inflammatory disorders and other reports have also pointed at PARP1 as a coactivator of the NF- $x \mathrm{~B}$ pathway (Hassa \& Hottiger, 2002).

Considering both conserved and divergent elements in mouse and human PHEX gene promoters (human PHEX proximal promoter region does not possess a long PolyA region characteristic for mice Phex; Fig. 1), we aimed to verify the cooperativity of PARP1 and NF$x \mathrm{~B}$ pathway in osteoblasts isolated from adult human bones $(\mathrm{HOb})$ as well as in cells derived from human osteosarcoma (MG-63).

PolyA

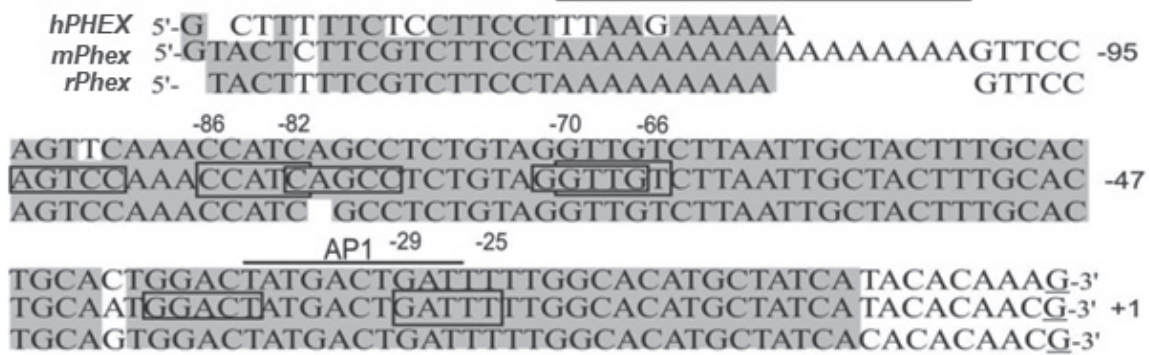

Figure 1. In silico analysis of the promoter regions of human PHEX ( $P$ PHEX), mouse Phex (mPhex) and rat Phex (rPhex).

The analysis included 133 nucleotides upstream the transcription start site $(+1)$. Nucleotide sequences in the brackets indicate the binding sites for the transcription factors of the NF-KB pathway proteins; the AP1 binding site and poly adenine-rich region (PolyA) are marked. 


\section{MATERIALS AND METHODS}

Chemicals and Reagents. Human osteoblast $(\mathrm{HOb}$, CAT 406-05a) were purchased from Cell Application INC (San Diego, CA, USA). Human osteosarcoma cells (MG-63, CAT: 86051601) and Osteoblast Growth Medium (CAT 417-500 CB) were purchased from ECACC Cell Lines (Salisbury, UK). The Antibiotic-Antimycotic solution, TRIzol reagent, TrypLE Express, DNA retardation gels, DMEM high glucose medium, Maxima First Strand cDNA Synthesis Kit for RT-qPCR, $2 \times \mathrm{Lu}-$ minaris Color Probe High ROX and T4 polynucleotide kinase were purchased from Thermo Fisher Scientific (Waltham, MA, USA). The Dual-Glo Luciferase Assay System, Caspase-Glo 3/7 Assay System, One Glo-Tox Kit, Gel Shift Binding $5 \times$ Buffer and NF- $x$ B consensus oligonucleotide were purchased from Promega (Madison, WI, USA). The TransIT-LT1 reagent was purchased from Mirus Bio LLC (Madison, WI, USA). TaqMan primer sets for real time RT-qPCR were purchased from Applied Biosystems (Foster City, CA, USA). [ $\left.\gamma^{32} \mathrm{P}\right] \mathrm{de}-$ oxyadenosine triphosphate $\left(\left[\gamma^{32} \mathrm{P}\right] \mathrm{dATP}\right)$ was purchased from Hartmann Analytic GmbH (Braunschweig, Germany). DNA oligonucleotides were synthesized by Genomed (Warsaw, Poland). Recombinant human TNF was purchased from PeproTech (Rocky Hill, NJ, USA). Fetal bovine serum was purchased from Biochrome GmbH (Berlin, Germany). The human expression plasmids pFLAG-CMV2-RELA and pFLAG-CMV2-NFKB1 were kindly provided by Dr. Christian Jobin (University of Florida, Gainesville). The Mini Quick Spin DNA Column kit was purchased from Roche (Basel, Switzerland), and the pNiFty2-Luc reporter vector was from InvivoGen (San Diego, CA. USA). All other reagents, unless otherwise indicated, were purchased from Sigma-Aldrich (St. Louis, MI, USA).

Cell culture. HOb and MG-63 cells were cultured in osteoblast growth medium (Cell Application INC, CAT 417-500 CB) or DMEM high glucose medium, containing $5 \%$ fetal bovine serum, 1\% Antibiotic-Antimycotic, $2 \mathrm{mM}$ L-glutamine and $3.4 \mathrm{mg} / 100 \mathrm{ml} \mathrm{L}$-ascorbic acid 2-phosphate sesquimagnesium salt, respectively. Cells were cultured at $37^{\circ} \mathrm{C}$ with $5 \% \mathrm{CO}_{2}$. For the analysis of PHEX gene expression, cells were seeded on 6-well plates (Becton Dickinson, Primaria) at $0.5 \times 10^{6}$ cells per well and were treated with TNF $(20 \mathrm{ng} / \mathrm{ml})$. In some experiments, the cells were pretreated for $30 \mathrm{~min}$ with the proteasome inhibitor clasto-lactacystin $\beta$-lactone $(\mathrm{cL} \beta \mathrm{L}, 10 \mu \mathrm{M})$ or the PARP1 inhibitor 3-aminobenzamide (3-AB,1 mM). Apoptosis and cytotoxicity were evaluated with the Caspase-Glo 3/7 Assay System and One Glo-Tox Kit, respectively, according to the manufacturers' protocols.

Real-Time RT-PCR analysis. One mg of total RNA isolated with TRIzol was reverse-transcribed using the Maxima First Strand cDNA Synthesis Kit for RT-qP$\mathrm{CR}$ and $25 \mathrm{ng}$ or $100 \mathrm{ng}$ of cDNA, regardless of experiments, were used for real time PCR analysis using commercially available primers and TaqMan probes (Hs 02758991_g1 for GAPDH and Hs 01011692_m1 for PHEX), Luminaris Color Probe qPCR Master Mix and the StepOne Real-Time PCR System. The resulting data were analyzed using the comparative cycle threshold $(\mathrm{Ct})$ method as means of relative quantification of gene expression, normalized to an endogenous reference glyceraldehyde-3-phospate dehydrogenase $(G A P D H)$ and relative to a calibrator (normalized $\mathrm{Ct}$ value obtained from control osteoblasts), and expressed as $2^{-\Delta \Delta} \mathrm{Ct}$ (Applied Biosystems User Bulletin number 2: Rev B "Relative Quantitation of Gene Expression”).
Transient transfections. $\mathrm{HOb}$ and MG-63 cells were seeded in 6 or 24 -well plates $\left(0.5 \times 10^{6}\right.$ or $0.5 \times 10^{5}$ cells/well, respectively), and cells were transfected with pNiFty2-Luc reporter vector $(1 \mu \mathrm{g} / \mathrm{ml}$ of medium) using the TransIT-LT1 transfection reagent $(3 \mu \mathrm{g} / \mathrm{ml})$. pNiFty2-Luc activity was estimated using Dual-Glo Luciferase Assay System and expressed as luciferase activity (RLU, relative light units) per $10 \mu \mathrm{l}$ of cell lysate. In some experiments, the cells were transfected with the expression vectors pFLAG-CMV2-RELA or/and pFLAG-CMV2NFKB1 $(0.5 \mu \mathrm{g} / \mathrm{ml}$ of medium), or the respective control pCMV2-null. After 15-24 h post transfection, the cells were used for total RNA isolation, and PHEX mRNA level was estimated as described in the "Real-Time RT-PCR Analysis" section.

Electrophoretic Mobility Shift Assay (EMSA). Nuclear protein (NP) for EMSA was prepared from the MG-63 cells as previously described (Slomiany, Kelly, \& Kurtz, 2000). Double-stranded, synthetic oligonucleotides were end-labeled with $\left[\gamma^{32} \mathrm{P}\right] \mathrm{dATP}$ and cleaned using Mini Quick Spin DNA Column, according to the manufacturers' protocols. For each reaction, $30000 \mathrm{cpm}$ of the probe were incubated at room temperature for 20 min with 5-10 $\mu \mathrm{g}$ of NP, $4 \mu \mathrm{l}$ of Gel Shift Binding $5 \times$ Buffer, $1 \mu \mathrm{g}$ of poly(d(I-C)), and $\mathrm{H}_{2} \mathrm{O}$ to a final volume of $20 \mu \mathrm{l}$. For competition studies, a 100 excess of unlabeled probe was added to the reaction. Antibodies for supershift assays (NFKB1, NFKB2, RELA and REL) were purchased from Santa Cruz Biotechnology (CAT: sc-7178, sc-298X, sc-372X and sc-272X, respectively). Binding reactions were loaded onto a $6 \%$ DNA retardation gel and were separated at 250 volts in 0.5 TBE. Gels were dried and then exposed to an X-ray film. The oligonucleotides used for the EMSA are depicted in Table 1.

Statistical analysis. The results were presented as mean values \pm S.E. for each parameter. Unless otherwise indicated, there were six observations in each group. Influence of TNF on mean levels of relative NF- $x \mathrm{~B}$ activation were compared using Welch two sample $t$-test for $\mathrm{HOb}$ and MG-63 cells separately. Two-way ANOVA was used to estimate effects of TNF and cL $\beta L, T N F$ and 3-AB, RELA and nuclear factor kappa B subunit 1 (NFKB1, synonym: p50) on relative expression of PHEX. Marginal tests were performed (type III sum of squares) and interaction between main factors was assessed. In all the tests we used significance level $\alpha=0.05$. Assumptions of normality and variance equality were checked using Shapiro-Wilk and Bartlett tests, respectively. The calculations were performed in $\mathrm{R}$ ver. 3.3.1 (R Core Team, 2016).

\section{RESULTS}

\section{TNF increase the NF-KB activity in human osteoblasts}

To confirm the activation of NF- $x \mathrm{~B}$ pathway under our experimental conditions, we transfected cells with

Table 1. Probe sequences used in EMSA presented in Fig. 7

\begin{tabular}{ll}
\hline Species: symbol & Probes used in EMSA studies \\
\hline Human: $h$ PHEX(1) & TTAAGAAAAAGT \\
\hline Human: $h$ PHEX(2) & TTAAGAAAAAGTCCAGTTC \\
\hline Rat: $r$ Phex & CTAAAAAAAAAGT \\
\hline Mouse: mPhex & CTAAAAAAAAAAAAAAAAAGT \\
\hline
\end{tabular}


A

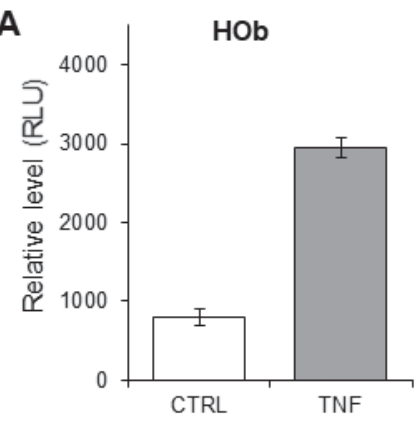

B

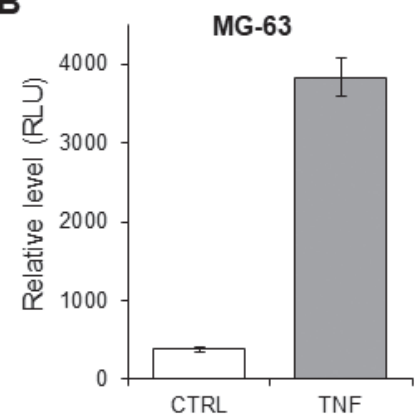

Figure 2. The activity of the NF-KB signaling pathway in the control (CTRL) and TNF treated HOb (A) or MG-63 (B) osteoblasts.

The charts show mean $(n=6) \pm$ standard error. Statistical significance is indicated in the results section.
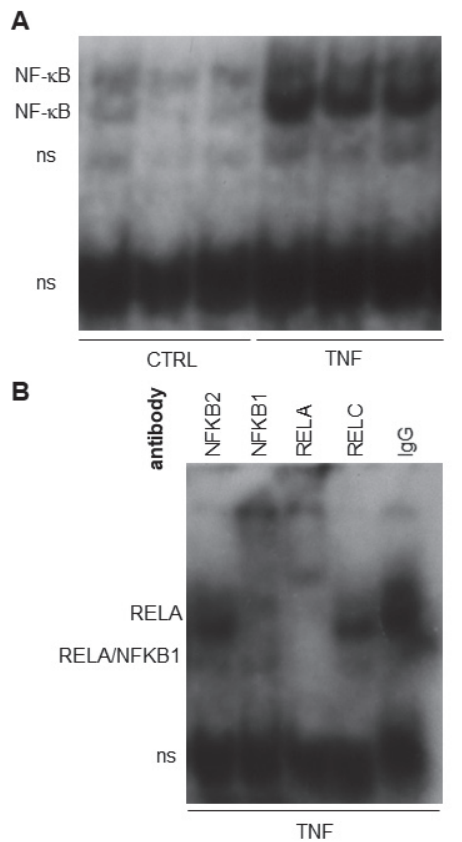

Figure 3. Electrophoretic mobility shift assay (EMSA) of the nuclear proteins isolated from MG-63 cells with (TNF) or without (CTRL) $24 \mathrm{~h}$ TNF treatment (A). Supershift/blocking EMSA analysis of the NF-KB/DNA complexes with extra anti-NF-KB pathway antibody (B).

The probe was NF-KB consensus (Promega, sequence: AGTTGAGGGGACTITCCCAGG). (A) The picture shows specific (NF-KB) and nonspecific (ns) migrating protein/DNA complexes. A longer electrophoresis and application of the anti-NF-KB antibody (B) enabled for identification of the main effectors of NF-KB pathway (RELA and NFKB1) migrating to the nuclei of the osteoblasts after TNF treatment.
NF- $x$ B-Luc luciferase reporter vector, pNiFty2-Luc. Cells were transfected in 24 well plates upon reaching $\sim 50 \%$ confluency, and the media were changed 15-24 h post-transfection to one without or with TNF (20 ng/ $\mathrm{ml}) .24$ hours later, the cells were lysed and analyzed for luciferase activity. The $t$-test analyses indicated significant increase of NF- $x \mathrm{~B}$ activity in TNF-treated $\mathrm{HOb}$ (Fig. 2A) and MG-63 osteoblasts (Fig. 2B) in comparison to untreated cells (CTRL) $(p<0.001 ; 95 \%$ confidence intervals 1769-2541 and 2821-4079, respectively). After TNF-treatment of $\mathrm{HOb}$ and MG-63 cells we found 4to 10 -fold increase in the activity of $\mathrm{NF}-x \mathrm{~B}$ pathway, respectively.

To identify the key components of the NF-xB complex activated by TNF, we performed electromobility shift assays (EMSA). MG-63 cells at $\sim 80 \%$ confluency were treated with TNF $(20 \mathrm{ng} / \mathrm{ml})$ and incubated for 24 hours. Nuclear protein (NP) was extracted from control and TNF treated osteoblasts as previously described (Slomiany et al., 2000). Double-stranded, commercially available $\mathrm{NF}-x \mathrm{~B}$ consensus oligonucleotides were end-labeled with $\left[\gamma^{32} \mathrm{P}\right]$ dATP and incubated with NP together with (Super shift) or without (EMSA) the antibodies recognizing individual $\mathrm{NF}-x \mathrm{~B}$ effector proteins. TNF induced a formation of protein/DNA complexes as shown in the picture (Fig. 3A). Incubation of NP isolated from TNF-treated osteoblasts with antibodies (Fig. 3B) demonstrated that complexes binding with NF- $x \mathrm{~B}$ consensus oligo consisted mainly of RELA (synonym: p65) and NFKB1 (synonym: p50). Supplementation of EMSA reaction with antibodies targeting nuclear factor kappa B subunit 2 (NFKB2, synonym: p52), REL proto-oncogene NF- $x \mathrm{~B}$ subunit (REL, synonym: c-REL), or control IgG did not affect protein/DNA complex formation, thus indicating that in response to TNF stimulation, human osteoblasts activate primarily the classical NFKB1/ RELA-dependent pathway.

\section{TNF-mediated PHEX inhibition is NF-KB-dependent}

The aim of this experiment was to study the mechanism of TNF influence on the PHEX gene expression. To this end, cells that achieved approximately $80 \%$ confluency in the cell culture were treated with TNF (20 $\mathrm{ng} / \mathrm{ml}$ ) and incubated for 24 hours. To test whether $\mathrm{NF}-x \mathrm{~B}$ activation is required for the inhibitory effects on the PHEX mRNA level, cells were pre-treated with clasto-lactacystin $\beta$-lactone (cL $\beta \mathrm{L}, 10 \mu \mathrm{M}$ ), an irreversible proteasome inhibitor. Proteasome inhibition stabilizes $\mathrm{I} \varkappa \mathrm{B} \alpha$ (nuclear factor of kappa light polypeptide gene enhancer in B-cells inhibitor alpha; NFKBIA), a natural inhibitor of nuclear translocation of the NF- $x \mathrm{~B}$ complex. For both $\mathrm{HOb}$ (Fig. 4A) and MG-63 (Fig. 4B), the average level of PHEX mRNA was significantly different from zero $(p<0.001)$ in the control groups. TNF significantly decreased the PHEX mRNA level compared to control for both HOb $(p<0.05)$ and MG-63 $(p<0.001)$. However, the effect of cL $\beta \mathrm{L}$ was cell line dependent: the level of PHEX mRNA increased significantly in $\mathrm{HOb}$ $(p<0.001)$ upon stimulation but remained the same in MG-63 cells. Yet, in MG-63 cells, we observed a strong interaction between TNF and cL $\beta \mathrm{L}(p<0.001)$, i.e. pretreatment with cL $\beta \mathrm{L}$ reversed the inhibitory effect of TNF on PHEX mRNA level, which increased by $46 \%$ in the cL $\beta \mathrm{L}$ and TNF pre-treated cells, compared to $87 \%$ in the cells pre-treated with TNF only.

To test the effects of RELA and NFKB1 proteins on PHEX expression, $\mathrm{HOb}$ and MG-63 cells were seeded in 6-well plates and transfected with pFLAG-CMV2- 

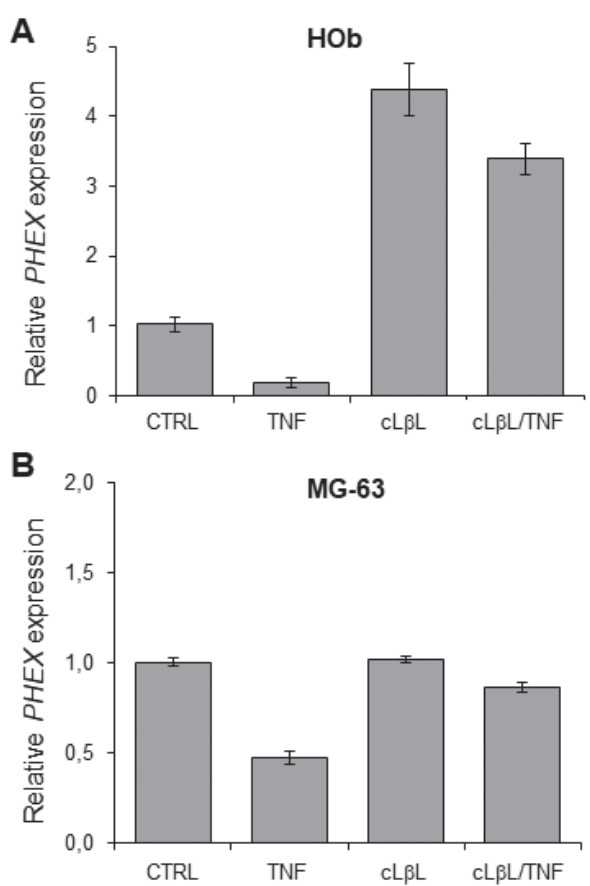

Figure 4. The relative level of Phex mRNA in (A) HOb and (B) MG-63 cells shown as mean $(n=5) \pm$ standard error.

The level of mRNA in the CTRL group was normalized to 1. Both control (CTRL) and TNF treated osteoblasts (TNF) were additionally pre-treated with the proteasome inhibitor clasto-Lactacystin $\beta$-lactone (cL $\beta L)$. Statistical significance is indicated in the results section.

RELA or/and pFLAG-CMV2-NFKB1, or the control vector pCMV2-null (CTRL). $24 \mathrm{~h}$ after transfection, total RNA was isolated and PHEX mRNA expression was estimated with RT-qPCR. The statistical analysis did not find any differences in PHEX mRNA level between control and either $\mathrm{HOb}$ (Fig. 5A) or MG-63 (Fig. 5B) cells, regardless of RELA, NFKB1 or NFKB1/RELA overexpression.

\section{PARylation inhibitor 3-AB does not reverse the effects of TNF on PHEX expression}

To test if PARP1 enzymatic activity modulates the effect of TNF $(20 \mathrm{ng} / \mathrm{ml}$ ) on the PHEX mRNA level, osteoblasts at $\sim 80 \%$ confluency were pre-treated with PARP inhibitor 3-aminobenzamide (3-AB, $1 \mathrm{mM})$. Both in $\mathrm{HOb}$ (Fig. 6A) and in MG-63 (Fig. 6B) cells, mRNA level of PHEX was significantly different from zero $(p<0.001)$ in the control groups. TNF treatment significantly decreased PHEX mRNA level, as compared to control, in both $\mathrm{HOb}(p<0.01)$ and MG-63 $(p<0.001)$. Pre-treatment with $3-\mathrm{AB}$ neither changed the level of PHEX mRNA when applied alone, nor reversed the inhibitory effect of TNF in both cell lines.

\section{Differential protein/DNA complex formation at the proximal mouse, rat, and human Phex/PHEX promoter after TNF treatment}

The aim of this experiment was to study the potential of species-specific Phex/PHEX proximal promoter segments in binding transcription factors using electromobility shift assay (EMSA). To this end, MG-63 cells that achieved approximately $80 \%$ confluency were treated with TNF $(20 \mathrm{ng} / \mathrm{ml})$ and incubated for 24 hours. The nuclear proteins (NP) was isolated and used for a
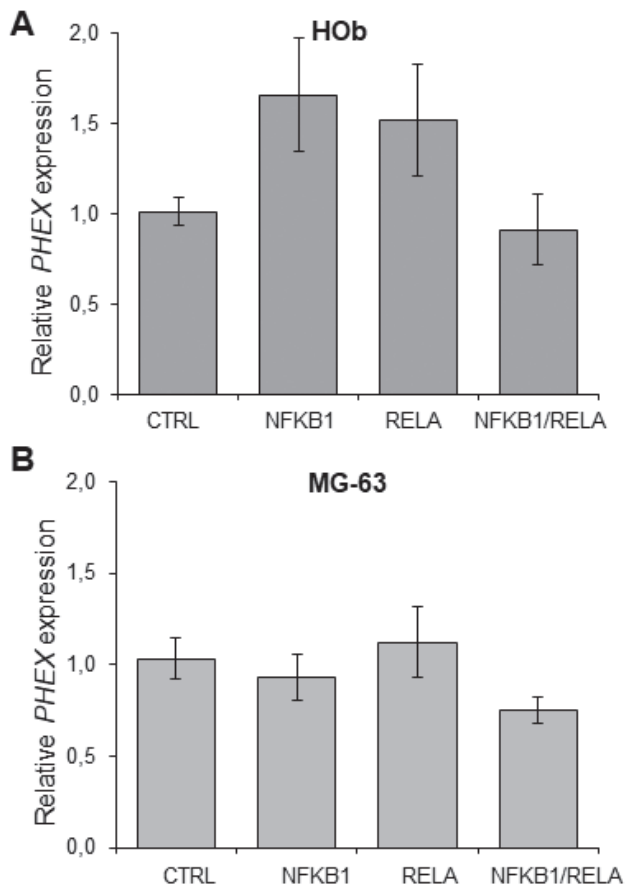

Figure 5. The relative level of Phex mRNA in (A) HOb and (B) MG-63 cells shown as mean $(n=6) \pm$ standard error.

The level of mRNA in the CTRL and cells overexpressing RELA, NFKB1 or both RELA and NFKB1 (group NFKB1/RELA). Overexpression was obtained by transfection of cells with expression plasmids pFLAG-CMV-RELA and pFLAG-CMV2-NFKB1. The level of mRNA in the control group (transfection with control plasmid $p C$ MV2-null) was normalized to 1 . Statistical significance is indicated in the results section.
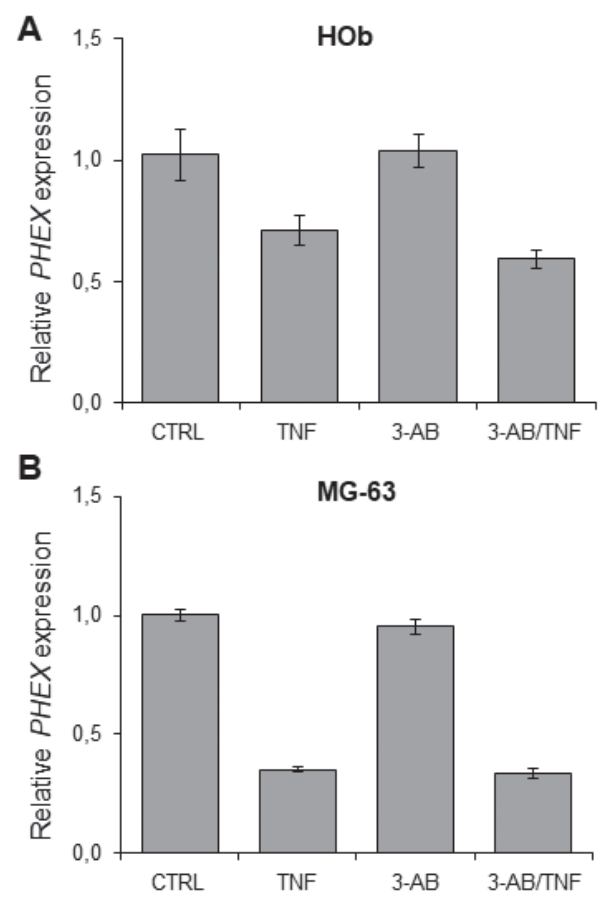

Figure 6. The relative level of Phex mRNA in (A) HOb and (B) MG-63 cells shown as mean $(n=6) \pm$ standard error.

The level of mRNA in the CTRL group was normalized to 1. Control (CTRL) and TNF treated osteoblasts (TNF) were additionally pre-treated with the inhibitor of poly(ADP) ribosylation (PARylation) 3-aminobenzamide (3-AB). Statistical significance is indicated in the results section. 


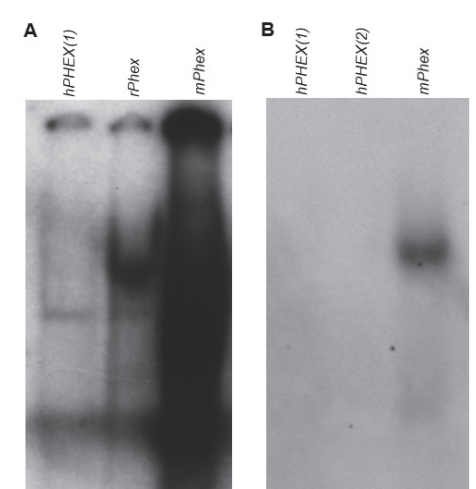

Figure 7. Electrophoretic mobility shift assay (EMSA) of the nuclear proteins isolated from TNF-treated MG-63 cells after $24 \mathrm{~h}$. (A) EMSA analysis of protein-DNA complexes with part of human ( $h P H E X)$, rat ( $r$ Phex) and mouse (mPhex) proximal promoter. (B) EMSA analysis of the protein-DNA complexes with two human PHEX(1), PHEX(2) promoters differing in length, and a mouse (mPhex) promoter. Probe sequences used in the EMSA are presented in Table 1.

test with double-stranded oligonucleotides that were complementary for analogous proximal promoter regions of mice (mPhex), rat (rPhex) and human (hPHEX), containing (mouse, rat) or not (human) adenine-rich regions (PolyA), as well as oligonucleotides complementary to different lengths of human PHEX promoter: hPHEX(1) and $h P H E X(2)$. The sequences are presented in Table 1. Double-stranded oligonucleotides were end-labeled with $\left[\gamma^{32} \mathrm{P}\right] \mathrm{dATP}$, incubated with NP $(10 \mu \mathrm{g})$, and divided on the retardation gel. We found that the length of the PolyA region in the promoters determined the level of migrating of the protein-DNA complexes (Fig. 7A). The EMSA signal was the highest for the mPhex promoter, lower for the rPhex promoter, and the hPhex promoter did not bind to any proteins (Fig. 7A). Comparison of the signal detected in EMSA coming from mPhex and the two different $h P H E X$ parts of the proximal promoter, additionally confirmed the capability of the PolyA region of binding NP (Fig. 7B). The EMSA signal was detected for the mPhex promoter, but both different hPHEX(1) and $h P H E X(2)$ parts of the promoters did not bind any proteins in the same experimental conditions.

\section{DISCUSSION}

We found that TNF reduces the expression of the human PHEX gen in both $\mathrm{HOb}$ and MG-63 osteoblasts, and these results are in concordance with those obtained after TNF treatment of a rat osteosarcoma cell line (UMR-106) (Majewski et al., 2010). Additionally, other published data coming from the rodent cells confirm this observation. Recently, it was shown that in differentiated mouse osteocyte-like IDG-SW3 cells, Phex mRNA levels decreased after treatment with the TNF, IL-1 $\beta$ or LPS (Ito et al., 2015). It should be noted that in the preliminary dose-response experiments (not shown), we had determined that it was necessary to use 2 to 4 higher concentration of TNF $(20 \mathrm{ng} / \mathrm{ml})$ to elicit the same effect on the PHEX expression in human osteoblasts, compared to the rat UMR-106 (10 ng/ml) or mouse IDG-SW3 (5 ng/ml) cells, respectively. Thus, we subsequently used this higher concentration of TNF in all the experiments conducted on $\mathrm{HOb}$ and MG-63 cells. The difference between the required dose in the tested cell systems may be related to species-specific expression of the TNF-receptors, and nonsignaling (decoy) TNF re- ceptors, or may be simply a unique characteristic of each cell line. It was previously confirmed that human osteoblasts derived from mesenchymal stem cells and MG-63 human osteosarcoma cells, used in our study, have similar complement of receptors for TNF (Bu et al., 2003).

Our data demonstrated that the $\mathrm{NF}-x \mathrm{~B}$ signaling pathway is involved in TNF-induced repression of human PHEX gene. Using the pNiFty2-Luc reporter vector with five $\mathrm{NF}-x \mathrm{~B}$ repeated transcription factor binding sites, we confirmed the increase in the NF- $x \mathrm{~B}$ signaling pathway activity in TNF-treated OBs. Moreover, we found that the inhibition of proteasome activity by pretreatment of OBs with cL $\beta \mathrm{L}$ reversed the effects of TNF on PHEX mRNA expression. These results strongly correspond with data derived from rat UMR-106 cells (Majewski et al., 2010). Interestingly, our data not only indicate that inhibition of $\mathrm{NF}-x \mathrm{~B}$ signaling pathway activity reversed the effect of $\mathrm{TNF}$, but also indicate that human PHEX can be under constitutive repression of this pathway. In $\mathrm{HOb}$ cells PHEX expression increased significantly after treatment with cL $\mathrm{L}$ in both control and TNF treated groups, when compared to the other groups. These effects were not visible in MG-63 cells. It is important to note that MG-63 cells are osteosarcoma cells derived from malignant bone tumors. They share some osteoblastic features (Declercq et al., 2004), but their chromosomal modifications lead to unusual cellular and molecular functions (Al-Romaih et al., 2003). While osteosarcoma-derived cells are generally used as osteoblastic models, their molecular malfunctions are not yet fully studied. Ultimately, the expression of extracellular matrix proteins contributing to the osteosarcoma-osteoid is changed, hence the differences compared to normal OBs (Benayahu, Shur, Marom, Meller, \& Issakov, 2001). In EMSA and supershift analyses, we identified $\mathrm{NF}-x \mathrm{~B}$ pathway components, which levels increased in OBs nuclei following the TNF treatment. EMSA from TNF-treated MG-63 cells with NF- $x \mathrm{~B}$ consensus probe showed an increased formation of $\mathrm{DNA} /$ protein complexes, which consisted predominantly of NFKB1 and RELA, highlighting the dominant role of its heterodimer in the response to TNF in human OBs. These data are consistent with our previous data derived from experiments conducted on rat UMR-106 cells (Majewski et al., 2010). However, in experiments with overexpression of NFKB1 and RELA in human OBs, we did not observe a suppressive effect on PHEX mRNA expression. This contrasts with the rat Phex expression, which was inhibited by the overexpression of both RELA alone and RELA with NFKB1 (Majewski et al., 2010).

Considering the previously reported importance of PARP1 in regulating Phex expression in rodents (Majewski et al., 2010), we investigated whether inhibition of PARP1 enzymatic activity by $3-\mathrm{AB}$ would alter the response to TNF. Interestingly, $3-\mathrm{AB}$ did not abrogate the effects of TNF on the expression of PHEX mRNA in the human osteoblasts in previously effective dose (1 $\mathrm{mM})$. It suggested that the role of PARP1 in the regulation of human PHEX promoter activity may be less important than in rodents.

Although PARP1 is an abundant and ubiquitous nuclear enzyme that was originally identified as a key factor in the DNA repair pathway, it has now been shown to positively and negatively affect gene transcription and chromatin structure under both basal and signal-activated conditions (Nguewa et al., 2005). Investigations of gene expression profiles in PARP1-deficient embryonic stem cells and in liver cells from PARP-1 KO mice demonstrated that $3.5 \%$ of the transcriptome was regulated by 
PARP1, as $2.4 \%$ were positively regulated (Ogino et al., 2007). Moreover, PARP1 has been described as one of the major molecules involved in the propagation of inflammatory stimuli and has been proposed as a target for anti-inflammatory treatment (Peralta-Leal et al., 2009). PARP1 has been shown to affect gene transcription in several ways: (1) it may act as an enhancer-binding factor similar to classical sequence-specific DNA-binding activators or repressors; (2) it may work as a transcriptional co-regulator or (3) it may act as a modifying enzyme that catalyzes the NAD-dependent addition of PARylation to numerous nuclear proteins. In the experiments conducted on mice and in UMR-106 cells, it was demonstrated that in TNF-treated OBs RELA is translocated to the nucleus, where it is PARylated by PARP1 (Majewski et al., 2010). This chemical modification is critical for TNF-induced inhibition of rodent Phex expression, and this response does not occur in PARP1-deficient mice and is reversed by the PARP1 inhibitor 3-AB or by overexpression of PARG (Majewski et al., 2010). According to this study, TNF treatment of UMR-106 resulted in binding of the NFKB1/RELA complex to the two sites located between the transcription start site and the PolyA region of the Phex gene promoter $(-76 /-57$ and $-35 /-16)$. The same region identified as the binding region for the NFKB1/RELA heterodimer was found in the human PHEX promoter; thus, it is possible that the human NFKB1/RELA heterodimer acts in the same way as in the rodent Phex promoter. However, additional investigations are necessary to confirm this. Using both molecular and proteomic methods, it was shown that PARP1 enzyme binds to the PolyA region in rodents and, under the influence of TNF, PARylates RELA subunits, thereby increasing their affinity for the proximal Phex promoter, and consequently inhibiting RNA polymerase II complex (Majewski et al., 2010). The human PHEX proximal promoter region does not possess a long PolyA region characteristic for mice Phex (Fig. 1). This difference in the promoter structures provides the opportunity to verify the hypothesis of the increased importance of PARP1, which has been demonstrated to act as a regular transcription factor that depends on proximal promoter organization. Current EMSA studies have shown that the number of adenines in the PolyA regions of promoters from three examined species is crucial for the level of protein interactions with this region of the gene. It seems to be very interesting whether other genes containing PolyA regions that are located close to the TSS region may be regulated by the described mechanism.

In conclusion, TNF inhibits PHEX gene expression in human OBs via a mechanism only partially consistent with rodent Phex gene. It probably requires a NFKB1/ RELA NF- $x \mathrm{~B}$ heterodimer, but it does not rely on PARP1 activity, as we previously reported for rat and mouse. Differences in the organization of the PHEX gene promoters and different requirements for the NF$x \mathrm{~B}$ complex likely explain these divergent mechanisms.

\section{Acknowledgements}

We would like to thank Yasmin Poniatowicz, Marta Lopacinska and Lukas Wojda, students from the Department of Animal Physiology, Faculty of Biology, University of Warsaw for their technical assistance.

\section{Acknowledgements of Financial Support}

This work was supported by National Science Centre grant N N401 629140.

\section{REFERENCES}

Al-Romaih K, Bayani J, Vorobyova J, Karaskova J, Park PC, Zielenska M, Squire JA (2003) Chromosomal instability in osteosarcoma and its association with centrosome abnormalities. Cancer Genet Cytogenet 144: 91-99. doi: 10.1016/S0165-4608(02)00929-9

Benayahu D, Shur I, Marom R, Meller I, Issakov J (2002) Cellular and molecular properties associated with osteosarcoma cells. I Cell Biochem 84: 108-114. doi: 10.1002/jcb.1270

Bowe AE, Finnegan R, Jan de Beur SM, Cho J, Levine MA, Kumar R, Schiavi SC (2001) FGF-23 inhibits renal tubular phosphate transport and is a PHEX substrate. Biochem Biophys Res Commun 284: 977-981. doi: 10.1006/bbrc.2001.5084

Bu RF, Borysenko CW, Li YN, Cao LH, Sabokbar A, Blair HC (2003) Expression and function of TNF-family proteins and receptors in human osteoblasts. Bone 33: 760-770. doi: 10.1016/j. bone.2003.07.006

Chang J, Wang Z, Tang E, Fan Z, McCauley L, Franceschi R, Wang CY (2009) Inhibition of osteoblastic bone formation by nuclear factor-kappaB. Nat Med 15: 682-689. https://doi.org/10.1038/nm.1954

Clowes JA, Riggs BL, Khosla S (2005) The role of the immune system in the pathophysiology of osteoporosis. Immunol Rev 208: 207-227. doi: $10.1111 / \mathrm{j} .0105-2896.2005 .00334 . x$

Collins JF, Ghishan FK (1994) Molecular cloning, functional expression, tissue distribution, and in situ hybridization of the renal sodium phosphate $(\mathrm{Na}+/ \mathrm{P}(\mathrm{i}))$ transporter in the control and hypophosphatemic mouse. FASEB J 8: 862-868. doi: 10.1096/fasebj.8.11.8070635

Declercq H, Van den Vreken N, De Maeyer E, Verbeeck R, Schacht E, De Ridder L, Cornelissen M (2004) Isolation, proliferation and differentiation of osteoblastic cells to study cell/biomaterial interactions: comparison of different isolation techniques and source. Biomaterials 25: 757-768. doi: 10.1016/S0142-9612(03)00580-5

Dixon PH, Christie PT, Wooding C, Trump D, Grieff M, Holm I, Thakker RV (1998) Mutational analysis of PHEX gene in X-linked hypophosphatemia. J Clin Endocrinol Metab 83: 3615-3623. doi: 10.1210/jcem.83.10.5180

Drezner MK (2000) PHEX gene and hypophosphatemia. Kidney Int 57: 9-18. doi: 10.1046/j.1523-1755.2000.00807.x

Du L, Desbarats M, Viel J, Glorieux FH, Cawthorn C, Ecarot B (1996) cDNA cloning of the murine Pex gene implicated in X-linked hypophosphatemia and evidence for expression in bone. Genomics 36: 22-28. doi: 10.1006/geno.1996.0421

Francis F, Korn SHB, Reinhardt R, de Jong P, Poustka A, Lehrach H, Rowe PSN, Goulding JN, Summerfield T, Mountford R, Read AP, Popowska E, Pronicka E, Davies KE, O’Riordan JLH, Econs MJ, Nesbitt T, Drezner MK, Oudet C, Pannetier S, Hanauer A, Strom TM, Meindl A, Lorenz B, Cagnoli B, Mohnike KL, Murken J, Meitinger T (1995) A gene (PEX) with homologies to endopeptidases is mutated in patients with X-linked hypophosphatemic rickets. The HYP Consortium. Nat Genet 11: 130-136. doi: 10.1038/ ng1095-130

Ghishan FK, Kiela PR (2011) Advances in the understanding of mineral and bone metabolism in inflammatory bowel diseases. Am I Physiol Gastrointest Liver Physiol 300: G191-201. doi: 10.1152/ajpgi.00496.2010

Hassa PO, Hottiger MO (2002) The functional role of poly(ADP-ribose)polymerase 1 as novel coactivator of NF-kappaB in inflammatory disorders. Cell Mol Life Sci 59: 1534-1553. doi: 10.1007/s00018$002-8527-2$

Hernando N, Biber J, Forster I, Murer H (2005) Recent advances in renal phosphate transport. Ther Apher Dial 9: 323-327. doi: 10.1111/j.1744-9987.2005.00290.x

Ito N, Wijenayaka AR, Prideaux M, Kogawa M, Ormsby RT, Evdokiou A, Atkins GJ (2015) Regulation of FGF23 expression in IDGSW3 osteocytes and human bone by pro-inflammatory stimuli. Mol Cell Endocrinol 399: 208-218. doi: 10.1016/j.mce.2014.10.007

Johnell O, Kanis J (2005) Epidemiology of osteoporotic fractures. Osteoporos Int 16 (Suppl 2): S3-S7. doi: 10.1007/s00198-004-1702-6

Johnell O, Kanis JA (2004) An estimate of the worldwide prevalence, mortality and disability associated with hip fracture. Osteoporos Int 15: 897-902. doi: 10.1007/s00198-004-1627-0

Johnell O, Kanis JA, Oden A, Sernbo I, Redlund-Johnell I, Petterson C, Jonsson B (2004) Mortality after osteoporotic fractures. Osteoporos Int 15: 38-42. doi: 10.1007/s00198-003-1490-4

Kiela PR, Ghishan FK (2009) Recent advances in the renal-skeletal-gut axis that controls phosphate homeostasis. Lab Invest 89: 7-14. doi: 10.1038/labinvest.2008.114

Krishnakumar R, Gamble MJ, Frizzell KM, Berrocal JG, Kininis M, Kraus WL (2008) Reciprocal binding of PARP-1 and histone H1 at promoters specifies transcriptional outcomes. Science 319: 819-821. doi: $10.1126 /$ science. 1149250

Larmonier CB, Shehab KW, Laubitz D, Jamwal DR, Ghishan FK, Kiela PR (2016) Transcriptional reprogramming and resistance to colonic mucosal injury in Poly(ADP-ribose) Polymerase 1 (PARP1)- 
deficient mice. J Biol Chem 291: 8918-8930. doi: 10.1074/jbc. M116.714386

Majewski PM, Thurston RD, Ramalingam R, Kiela PR, Ghishan FK (2010) Cooperative role of NF-\{kappa $\}$ B and poly(ADP-ribose) polymerase 1 (PARP-1) in the TNF-induced inhibition of PHEX expression in osteoblasts. J Biol Chem 285: 34828-34838. doi: 10.1074/jbc.M110.152868

Murer H, Hernando N, Forster I, Biber J (2003) Regulation of Na/ Pi transporter in the proximal tubule. Annu Rev Physiol 65: 531-542. doi: 10.1146/annurev.physiol.65.042902.092424

Nguewa PA, Fuertes MA, Valladares B, Alonso C, Perez JM (2005) Poly(ADP-ribose) polymerases: homology, structural domains and functions. Novel therapeutical applications. Prog Biophys Mol Biol 88: 143-172. doi: 10.1016/j.pbiomolbio.2004.01.001

Novack DV (2011) Role of NF-kappaB in the skeleton. Cell Res 21: 169-182. doi: 10.1038/cr.2010.159

Ogino H, Nozaki T, Gunji A, Maeda M, Suzuki H, Ohta T, Masutani M (2007) Loss of Parp-1 affects gene expression profile in a genome-wide manner in ES cells and liver cells. BMC Genomics 8: 41. doi: 10.1186/1471-2164-8-41

Peralta-Leal A, Rodriguez-Vargas JM, Aguilar-Quesada R, Rodriguez MI, Linares JL, de Almodovar MR, Oliver FJ (2009) PARP inhibitors: new partners in the therapy of cancer and inflammatory diseases. Free Radic Biol Med 47: 13-26. doi: 10.1016/j.freeradbiomed.2009.04.008
R Core Team (2016) R: A language and environment for statistical computing. R Foundation for Statistical Computing, Vienna, Austria. https://www.R-project.org/

Rodriguez-Bores L, Barahona-Garrido J, Yamamoto-Furusho JK (2007) Basic and clinical aspects of osteoporosis in inflammatory bowel disease. World J Gastroenterol 13: 6156-6165. doi: 10.3748/wig.v13. i46.6156

Slomiany BA, Kelly MM, Kurtz DT (2000) Extraction of nuclear proteins with increased DNA binding activity. Biotechniques 28: $938-942$

Sponholtz TR, Zhang X, Fontes JD, Meigs JB, Cupples LA, Kiel DP, McLean RR (2014) Association between inflammatory biomarkers and bone mineral density in a community-based cohort of men and women. Arthritis Care Res (Hoboken) 66: 1233-1240. doi: 10.1002/ acr. 22270

Teti A, Zallone A (2009) Do osteocytes contribute to bone mineral homeostasis? Osteocytic osteolysis revisited. Bone 44: 11-16. doi: 10.1016/j.bone.2008.09.017

Uno JK, Kolek OI, Hines ER, Xu H, Timmermann BN, Kiela PR, Ghishan FK (2006) The role of tumor necrosis factor alpha in down-regulation of osteoblast Phex gene expression in experimental murine colitis. Gastroenterology 131: 497-509. doi: 10.1053/j.gastro.2006.05.020

Viswanathan A, Sylvester FA (2008) Chronic pediatric inflammatory diseases: effects on bone. Rev Endocr Metab Disord 9: 107-122. doi: 10.1007/s11154-007-9070-0 